\title{
Ciclos circadianos desajustados en la relación del consumo de alcohol y depresión
}

\author{
Juan Manuel Quijada Gaytán', Marcela Martinelli Herrera², Norberto Hernández Llanes² \\ I Director General de los Servicios de Atención Psiquiátrica, Secretaría de Salud \\ ${ }^{2}$ Comisión Nacional contra las Adicciones (CONADIC)
}

El consumo de bebidas embriagantes ha acompañado a las mujeres y los hombres a lo largo de la historia; el sentirse tristes en extremo, también. Muchas veces ambas situaciones se combinan y se crea algo difícil de sobrellevar.

Las mujeres y los hombres están regidos por cuestiones diversas que los determinan y sobrepasan, desde lo más simple, que es estar vivo, y en la mayoría de los casos llegar a la muerte. En el recorrido de lo que se puede llamar "estar y ser en el mundo", aparecen múltiples cuestiones que son decididas a voluntad, por mencionar algunas: qué se estudia, con quién se decide formar pareja y en algunos casos una familia, y así se podrían dar varios ejemplos. Aunque lo anterior puede ser considerado cierto, para algunos hasta esas cuestiones están determinadas por factores que los sujetos ni siquiera saben que existen.

Si se considera lo que se conoce como el reloj biológico de los seres vivos, se podría observar que existe una gran cantidad de procesos que acontecen en el cuerpo humano, los cuales son posibilitados por lo que regula y programa las funciones fisiológicas del organismo en periodos de 24 horas: ciclos circadianos. La etimología de la palabra circadiano deriva del latín circa que indica "aproximación", dies que significa "día" y el sufijo -anus, que se refiere a "algo relacionado con" (Ciclo circadiano, s.f.). Que sea un ciclo significa que se repite, en este caso día tras día, lo que en la biología permite la aparición de fenómenos fisiológicos como la presencia de diversas hormonas; por ejemplo, en la noche se incrementa la producción de melatonina, que se asocia con la facilitación del dormir. O en las plantas los ciclos circadianos permiten la aparición de la fotosíntesis, el crecimiento celular, entre otras cuestiones.

Lo anterior es posible, a su vez, por los ciclos de luz y oscuridad que caracterizan el día y la noche, lo cual permite que los humanos tengan ritmos circadianos, es decir, a las actividades que día con día se realizan y se vuelven hábitos, costumbres, cosas que se hacen sin pensar de- masiado en ello. A saber: levantarse, ingerir alimentos, asearse, realizar las actividades propias: estudio, trabajo y demás; por la tarde-noche cenar, prepararse para dormir y al día siguiente comienza de nuevo otro ciclo.

Pero, ¿qué sucede cuando lo anterior se ve trastocado? Cuando el día y la noche no son parte del devenir por la vida, sino una carga pesada con la que hay que lidiar. Ahí, los hábitos son retos a cumplir, la costumbre lastima y a veces duele tanto que se trata de evitar. Los límites entre el día y la noche se pueden ir borrando, o todo se vuelve un día eterno a atravesar, como un río caudaloso; o es una noche interminable poblada de seres que atemorizan.

Utilizar la metáfora del ciclo circadiano y su desajuste permite abordar lo que este año se propuso como lema para el Día Nacional contra el Uso Nocivo de Bebidas Alcohólicas: "Hablemos de alcohol y depresión". El 8 de mayo de 2018 se publicó en el Diario Oficial de la Federación (Secretaría de Gobernación [SEGOB], 2018) el decreto por el que se declara de manera oficial al 15 de noviembre de cada año para conmemorar este evento.

El alcohol es la principal sustancia psicoactiva consumida en México, ya que siete de cada 10 mexicanos lo han usado alguna vez en su vida, cuatro de cada 10 bebieron en el último mes y tres de cada 10 lo hicieron de forma excesiva en el último mes (el consumo excesivo de alcohol se define como el consumo de cinco o más copas estándar en el caso de hombres o cuatro copas o más por ocasión en el caso de las mujeres, entendiendo por copa estándar aquella bebida alcohólica que contiene 13 gramos de alcohol puro; (Instituto Nacional de Psiquiatría Ramón de la Fuente Muñiz [INPRFM], Instituto Nacional de Salud Pública [INSP], Comisión Nacional contra las Adicciones [CONADIC], Secretaría de Salud [SSA], 2017); su uso nocivo es uno de los importantes factores de riesgo a nivel mundial (Global Burden of Disease Collaborators, 2018), ya que su consumo es causa de 60 enfermedades y contribuye de manera importante a más de 200 problemas de salud (Organización Mundial de la

\footnotetext{
Autor de Correspondencia:

Juan Manuel Quijada Gaytán. Director General de los Servicios de Atención Psiquiátrica

Correo electrónico: jm.quijada@salud.gob.mx

doi: 10.28931/riiad.2020.2.07
} 
Salud [OMS], 2018). En la mayoría de los países es legal para mayores de edad, sin embargo, en menores de edad ya es un problema de salud, tanto en México como a nivel mundial. Los trastornos mentales y comportamentales tienen una relación causal con el consumo nocivo de alcohol. La depresión es una enfermedad mental, que en muchos casos está en íntima relación con el consumo de riesgo de bebidas alcohólicas.

Las razones por las que un sujeto consume alcohol de manera perjudicial para su salud física y mental, así como por qué alguien está sumido en una profunda depresión son particulares, aunque hay cuestiones estudiadas que permiten delimitar aspectos que se conocen como factores de riesgo, que pueden propiciar la aparición del consumo nocivo y de la depresión. Algunas de ellas son: las frustraciones e insatisfacciones de la vida diaria experimentadas por un periodo largo de tiempo; problemas económicos; pérdida del empleo; acontecimientos vitales como la pérdida de un ser querido o la ruptura de una relación sentimental; alguna enfermedad crónica; cambios hormonales (embarazo, menopausia); violencia intrafamiliar. Todos los factores anteriores influyen para que un individuo pueda llevar a cabo un ritmo de vida "normal". A continuación, se abordarán algunas dificultades de salud que se presentan en lo que se conoce como una patología dual, en este caso la interrelación del consumo nocivo de alcohol y la depresión.

El metabolismo del cerebro sufre afectaciones cuando se ingiere alcohol y cuando se está deprimido; lo curioso es que mucha gente cree que el alcohol le permite salir de su tristeza profunda. Lo anterior se debe a que, al comenzar a tomar, los efectos que se producen dan la falsa creencia de ser estimulantes y con eso se vive momentáneamente euforia, desinhibición y a veces hasta se puede llegar a olvidar el dolor que se tiene. Pero esto, como viene se va y a veces más rápido de lo que se desea, ya que el alcohol al ser un depresor del sistema nervioso central, como su nombre lo indica, deprime una serie de funciones lo que da como resultado que los malestares que se trataban de lubricar y hasta hacer desaparecer con el consumo de alcohol, vuelvan a experimentarse, y a veces con más fuerza y generando mayor malestar, ya que llevan consigo lo efectos adversos que el alcohol genera en el funcionamiento mental: distorsión de la realidad, olvidos, decir y realizar cosas que lastiman a otros y al mismo sujeto.

El ciclo circadiano se desajusta y altera, así como se puede perder la noción del tiempo, se presentan síntomas adversos como insomnio o exceso de dormir, los alimentos se rechazan por el malestar físico que se vive o se alteran radicalmente los horarios de su ingesta. Actividades cotidianas placenteras se convierten en acciones que generan angustia. La noche se tiñe de pesadillas vívidas sin necesidad de estar dormidos, los monstruos se presentifican sin que haya otro delante; las obligaciones cotidianas se vuelven piedras que aplastan y hunden más a los sujetos.

Estudios realizados en México muestran que la depresión es un trastorno frecuente, $4.5 \%$ de la población la padece (Belló et al., 2005) y 30\% de la población presenta sintomatología depresiva (Instituto Nacional de Estadística y Geografía [INEGI], 2017) que produce mayor discapacidad que otras condiciones crónicas como la diabetes, los trastornos respiratorios, las enfermedades cardiacas o la artritis. Las personas con depresión pierden hasta 25.51 días de trabajo al año en comparación con otro tipo de enfermedades crónicas (6.8 días aproximadamente).

La co-ocurrencia de trastornos depresivos y trastornos por uso de alcohol se asocia con mayor severidad y peor pronóstico de los mismos (McHugh \& Weiss, 2019), por lo que es importante que los servicios de salud implementen acciones de detección temprana, derivación y el tratamiento oportuno para ambas condiciones médicas.

Aristóteles plantea que: "Así pues, el vino tomado en abundancia parece que predispone a los hombres a caer en un estado semejante al de aquellos que hemos definido como melancólicos, y su consumo crea una gran diversidad de caracteres" (Aristóteles, 2007). De la euforia se pasa a la tristeza, de la valentía al miedo, de las ganas de vivir a pensar en el suicidio.

Una cita de Tomas De Quincey, escritor británico del romanticismo (Martínez Cortés, 2013) quien fue fumador de opio y bebedor de grandes cantidades de láudano y alcohol, ejemplifica cómo el alcohol u otras sustancias no libera a los sujetos de sus pesares:

"La mente no es capaz de nada que se parezca al olvido; mil accidentes interponen un velo entre nuestra conciencia y las inscripciones secretas de la mente, pero otros accidentes de la misma clase lo desgarran y, velado o no, la inscripción perdura para siempre como las estrellas que parecen retirarse ante la luz del día, aunque en verdad, como todos sabemos, la luz solamente haya corrido su velo sobre ellas, y volverán a mostrarse cuando otra vez se descorra la oscurecedora luz del día."

El consumo de alcohol puede hacer que se olvide por momentos la tristeza, o por el contrario, los sujetos pueden ahondar en ésta. De ahí que es tan importante prestar oídos a lo que los sujetos están diciendo con sus actos, sean estos dormir en exceso, una apatía devastadora o un consumo nocivo de alcohol que vuelve tóxico al sujeto y a su alrededor. Para escuchar a alguien hay que permitirle hablar: "Hablemos de alcohol y depresión", el lema 
de este año, abre la posibilidad de hacer intervenciones en distintos niveles, de prevención, reducción del daño o los daños y de tratamiento. Con lo anterior se posibilitará que los ciclos vitales vuelvan a ser eso, ciclos de vida, con sus días y sus noches, sus luces y oscuridades, incluyendo toda la paleta de colores que ofrece la vida y no permitir que las mujeres y los hombres se queden en la ceguera desoladora o se ahoguen en un vaso de alcohol.

\section{REFERENCIAS}

Aristóteles. (2007). El hombre de genio y la melancolía. Acantilado. Belló, M., Puentes-Rosas, E., Medina-Mora, M. E., Lozano, R. (2005) Prevalence and diagnosis of depression in Mexico. Salud Pública de México, 47(Supl.1), S4-S11

Ciclo circadiano. (s.f.). En Significados.com. https://www.significados.com/ciclo-circadiano

Global Burden of Disease Collaborators. (2018). Alcohol use and burden for 195 countries and territories, 1990-2016: a systematic analysis for the Global Burden of Disease Study 2016. The Lancet, 392(10152), 1015-1035. doi 10.1016/S01406736(18)31310-2
Instituto Nacional de Estadística y Geografía. (2017). Integrantes del hogar de 12 años y más con sentimientos de depresión. Encuesta Nacional de los Hogares. https://www.inegi.org.mx/ temas/salud/

Instituto Nacional de Psiquiatría Ramón de la Fuente Muñiz, Instituto Nacional de Salud Pública, Comisión Nacional contra las Adicciones, Secretaría de Salud. (2017). Encuesta Nacional de Consumo de Drogas, Tabaco y Alcohol 2016-2017: Reporte de Alcohol. Instituto Nacional de Psiquiatría Ramón de la Fuente Muñiz.

Martínez Cortés, F. (2013). La Epidemia Baudeleriana: Los Factores Psicosociales y Culturales de la Drogadicción. Art Grafitti Sistema Gráfico

McHugh, R. K. \& Weiss, R. D. (2019). Alcohol Use Disorder and Depressive Disorders. Alcohol research: current reviews, 4O(1), arcr.v40.1.01. doi:10.35946/arcr.v40.1.01.

Organización Mundial de la Salud. (2019). Global status report on alcohol and health, 2018. Editor.

Secretaría de Gobernación. (2018). Decreto por el que se declara el 15 de noviembre de cada año como "Día Nacional Contra el Uso Nocivo de Bebidas Alcohólicas". Diario Oficial de la Federación. http://dof.gob.mx/nota_detalle.php?codigo=5521802\&fecha $=08 / 05 / 2018$. 